\title{
Efficacy of isothiocyanate-based compounds on different forms of persistent pain
}

This article was published in the following Dove Press journal: Journal of Pain Research

\section{Elena Lucarini' \\ Laura Micheli' \\ Alma Martelli ${ }^{2}$ \\ Lara Testai ${ }^{2}$ \\ Vincenzo Calderone ${ }^{2}$ \\ Carla Ghelardini' \\ Lorenzo Di Cesare \\ Mannelli'}

'Pharmacology and Toxicology Section, Department of Neuroscience, Psychology, Drug Research, and Child Health (Neurofarba), University of Florence, Florence, Italy; ${ }^{2}$ Department of Pharmacy, University of Pisa, Pisa, Italy
Correspondence: Lorenzo Di Cesare Mannelli

Pharmacology and Toxicology Section, Department of Neuroscience, Psychology, Drug Research, and Child Health (Neurofarba), University of Florence, 6 Viale Pieraccini, Florence 50139, Italy

Tel +39055 2758395

Email lorenzo.mannelli@unifi.it
Purpose: Current pharmacotherapy for persistent pain related to neuropathy or articular diseases is unsatisfactory, due to the large number of unresponsive patients and side effects. Isothiocyanates (ITCs) are a class of natural or synthetic compounds characterized by the general formula R-NCS. ITCs show antihyperalgesic effects in models of central and peripheral nervous tissue injury and anti-inflammatory properties. The pharmacodynamics are strictly related to the release of the gasotransmitter hydrogen sulfide $\left(\mathrm{H}_{2} \mathrm{~S}\right)$ from their moiety. In particular, phenyl ITC (PITC) and 3-carboxyphenyl ITC (3C-PITC) exhibit interesting slow $\mathrm{H}_{2} \mathrm{~S}$-release properties suitable for treating painful pathology. The aim of the present work was to evaluate the efficacy of PITC and 3C-PITC against mechanical hyperalgesia and spontaneous pain induced by nerve injury and osteoarthritis.

Methods: Nerve injury and osteoarthritis were induced in rats by ligation of the sciatic nerve (chronic constriction injury) and intra-articular injection of monoiodoacetate, respectively. Behavioral tests were performed 14 days after damage induction.

Results: Single subcutaneous administrations of PITC, 3C-PITC (4.43 and $13.31 \mu \mathrm{mol} \mathrm{kg}{ }^{-1}$, respectively) were able to completely reverse hypersensitivity to noxious stimuli in both models of neuropathic and osteoarticular pain. The effect of ITCs was compared with that of NaHS, the prototypical $\mathrm{H}_{2} \mathrm{~S}$ donor, showing similar efficacy and higher potency. ITCs and NaHS also reduced spontaneous pain.

Conclusion: ITCs offer a promising novel approach to counteract persistent, drug-resistant painful pathology.

Keywords: neuropathic pain, nerve injury, osteoarthritis, isothiocyanate, $\mathrm{H}_{2} \mathrm{~S}$

\section{Introduction}

Persistent pain is a grave and debilitating condition that affects approximately a quarter of the population and is often associated with a considerable reduction in quality of life. ${ }^{1}$ There are many forms of persistent pain. Neuropathy- and arthritis-related pain are among the prevalent causes. ${ }^{2,3}$ Neuropathy results from central or peripheral nervous system damages. ${ }^{4}$ Pharmacological therapy for neuropathic pain still shows limited efficacy, combined with several side effects that limit increases in dosage. ${ }^{5}$ Osteoarthritis is a multifactorial joint disease, and includes joint degeneration, intermittent inflammation, and peripheral neuropathy, so it is characterized by persistent pain with both inflammatory and neuropathic components. ${ }^{6,7}$ The joint erosion also impairs local sensory afferents, leading to sensitization of the peripheral nervous system which may result in central augmentation of nociceptive processing. ${ }^{7}$ As consequence 
of severe osteoarthritis, an increasing number of patients exhibit neuropathic pain-like symptoms. ${ }^{6}$ In osteoarthritic patients, anti-inflammatory drugs are the first choice in the treatment of pain, despite their limited efficacy and several side effects, particularly in the elderly. ${ }^{8}$ The development of novel therapeutics for an effective and safe treatment of these types of pain becomes necessary. Isothiocyanates (ITCs) are a class of natural or synthetic compounds with the general formula R-NCS. ${ }^{9}$ Natural ITCs derive from the hydrolysis of glucosinolates, secondary metabolites mainly present in plants belonging to the family of Brassicaceae. ${ }^{10}$ Glucosinolates and ITCs show beneficial effects in models of central and peripheral nervous tissue injury, as well as in carcinogenesis and cardiovascular diseases. ${ }^{11-16}$ Moreover, ITCs exhibit anti-inflammatory properties as they act on the regulation of $\mathrm{NF} \kappa \mathrm{B}$, the modulation of $\mathrm{TNF} \alpha$, and the lipopolysaccharide-stimulated inflammatory response. ${ }^{17-20}$ Recently, we demonstrated the efficacy of subcutaneous (SC) and intracerebroventricular administration of different ITCs in a mouse model of chemotherapy-induced neuropathic pain. ${ }^{21}$ According to previously reported in vivo data, ${ }^{21}$ we found that the pharmacodynamics were closely related to the release of the gasotransmitter $\mathrm{H}_{2} \mathrm{~S} .{ }^{16}$ Among all natural and synthetic ITCs, phenyl ITC (PITC) and 3-carboxyphenyl ITC (3C-PITC) are endowed with interesting slow $\mathrm{H}_{2} \mathrm{~S}$-release properties that make them suitable for pharmacological applications. ${ }^{16,22}$ Indeed, PITC and 3C-PITC possess an optimal efficacy/potency profile in relieving chemotherapyinduced neuropathic pain. ${ }^{21}$ The aim of the present work was to evaluate the efficacy of PITC and 3C-PITC in reducing mechanical hyperalgesia and spontaneous pain in two rat models of persistent pain: traumatic injury to the sciatic nerve (chronic constriction injury [CCI]) and chemical erosion of the tibiotarsal joint (monoiodoacetate [MIA]). ${ }^{23,24}$ The efficacy of ITCs was also compared with the prototypical $\mathrm{H}_{2} \mathrm{~S}$ donor NaHS. ${ }^{25}$

\section{Methods}

\section{Animals}

Male Sprague Dawley rats (Envigo, Huntingdon, UK) weighing approximately 200-250 g were employed. Animals were housed in the Centro Stabulazione Animali da Laboratorio, University of Florence and used at least 1 week after their arrival. Four rats were housed per cage $(26 \times 41 \mathrm{~cm})$ kept at $23^{\circ} \mathrm{C} \pm 1^{\circ} \mathrm{C}$ with a 12 -hour light-dark cycle (light at 7 am), and were fed a standard laboratory diet and tap water ad libitum. All animal manipulations were carried out according to directive 2010/63/EU of the European Parliament and European
Union Council (September 22, 2010) on the protection of animals used for scientific purposes. The ethical policy of the University of Florence complies with the Guide for the Care and Use of Laboratory Animals of the US National Institutes of Health (NIH publication 85-23, revised 1996; University of Florence assurance number A5278-01). Formal approval to conduct the experiments described was obtained from the Animal Subjects Review Board of the University of Florence. Experiments involving animals have been reported according to ARRIVE (animal research: reporting in vivo experiments) guidelines. ${ }^{26}$ All efforts were made to minimize animal suffering and the number of animals used.

\section{$\mathrm{CCl}$-induced neuropathic pain model}

Neuropathy was induced according to the procedure described by Bennett and Xie. ${ }^{23}$ Briefly, rats were anesthetized with $2 \%$ isoflurane. Under aseptic conditions, the right (ipsilateral) common sciatic nerve was exposed at the level of the middle thigh by blunt dissection. Proximal to the trifurcation, the nerve was freed carefully from the surrounding connective tissue, and four chromic cat-gut ligatures (4-0; Ethicon, Somerville, NJ, USA) were tied loosely around the nerve with about $1 \mathrm{~mm}$ spacing between ligatures. After hemostasis had been confirmed, the incision was closed in layers. Animals were allowed to recover from surgery and then housed one per cage with free access to water and standard laboratory chow. Control rats were subjected to sham surgery in which the sciatic nerve was only exposed, but not ligated. Behavioral measurements were performed on day 14 after ligation.

\section{MIA-induced osteoarthritis model}

Unilateral osteoarthritis was also induced by injection of MIA (Sigma-Aldrich, St Louis, MO, USA) into the knee joint. ${ }^{24}$ On day 1 , rats were anesthetized slightly with $2 \%$ isoflurane, following abolition of the hind-paw pinch-withdrawal reflex, a 27-gauge needle was introduced into the joint cavity between the tibial plateau and femoral condyles. MIA (2 mg) in 25 $\mu \mathrm{L}$ saline was delivered into the left articular cavity. Control rats were treated with an equal volume of saline. Behavioral measurements were performed on day 14 .

\section{Compound administrations}

PITC (Sigma-Aldrich) and 3C-PITC (Fluorochem, Hadfield, UK) were dissolved in saline solution with $0.5 \%$ polyethylene glycol. Sodium hydrosulfide hydrate (NaHS; Sigma-Aldrich) was dissolved in saline solution. Acute measures were performed after SC administration of 4.43 and $13.31 \mu \mathrm{mol} \mathrm{kg} \mathrm{kg}^{-1}$ PITC, 3C-PITC, and NaHS. 


\section{Paw-pressure test}

The nociceptive threshold in each rat was determined with an analgesimeter (Ugo Basile, Gemonio, Italy) according to the method described by Leighton et al. ${ }^{27}$ Briefly, a constantly increasing pressure was applied to a small area of the dorsal surface of the paw using a blunt conical probe with a mechanical device. Mechanical pressure was increased until a withdrawal reflex occurred while rats were lightly restrained. Vocalization or withdrawal-reflex thresholds are expressed in grams. Rats scoring $<40 \mathrm{~g}$ or $>80 \mathrm{~g}$ during the test before drug administration were rejected (25\%).

\section{Incapacitance test}

Weight-bearing changes were measured using an incapacitance apparatus (Linton Instrumentation, Diss, UK) detecting changes in postural equilibrium after a hind-limb injury. ${ }^{28}$ Rats were trained to stand on their hind paws in a box with an inclined plane ( $65^{\circ}$ from horizontal). This box was placed above the incapacitance apparatus. This allowed us independently to measure the weight that the animal applied on each hind limb. The value considered for each animal was the mean of five consecutive measurements. In the absence of hind-limb injury, rats applied an equal weight on both hind limbs, indicating postural equilibrium, whereas an unequal distribution of the weight on hind limbs indicated a monolaterally decreased pain threshold. Data are expressed as the difference between the weight applied on the limb contralateral to the injury and the weight applied on the ipsilateral one ( $\Delta$ weight).

\section{Statistical analysis}

Behavioral measurements were performed on ten rats for each treatment carried out in two different experimental sets. All experimental procedures were performed by a researcher blind to the treatment. Standard ANOVA followed by Fisher's protected least significant difference procedure were used. Results are expressed as mean \pm standard error of the mean (SEM). All assessments were made by researchers blinded to rat treatments. Data were analyzed using Origin 9.1 (OriginLab, Northampton, MA, USA).

\section{Results}

Animals with monolateral peripheral neuropathy show an amplified response to mechanical stimuli on the ipsilateral paw and postural balance impairments related to the presence of ongoing pain. On day 14 after right sciatic nerve ligation, the weight tolerated on the ipsilateral paw was significantly lower (43.3 $\pm 3.3 \mathrm{~g}, \mathrm{CCI}+$ vehicle) in respect of sham-operated animals
$(68.3 \pm 3.1 \mathrm{~g}$, sham + vehicle; Figure 1A). The effects of a single SC administration of two different doses of PITC, 3C-PITC and NaHS on CCI-induced mechanical hypersensitivity (measured by paw-pressure test) are shown in Figure 1. Both doses of PITC completely abolished mechanical hypersensitivity in
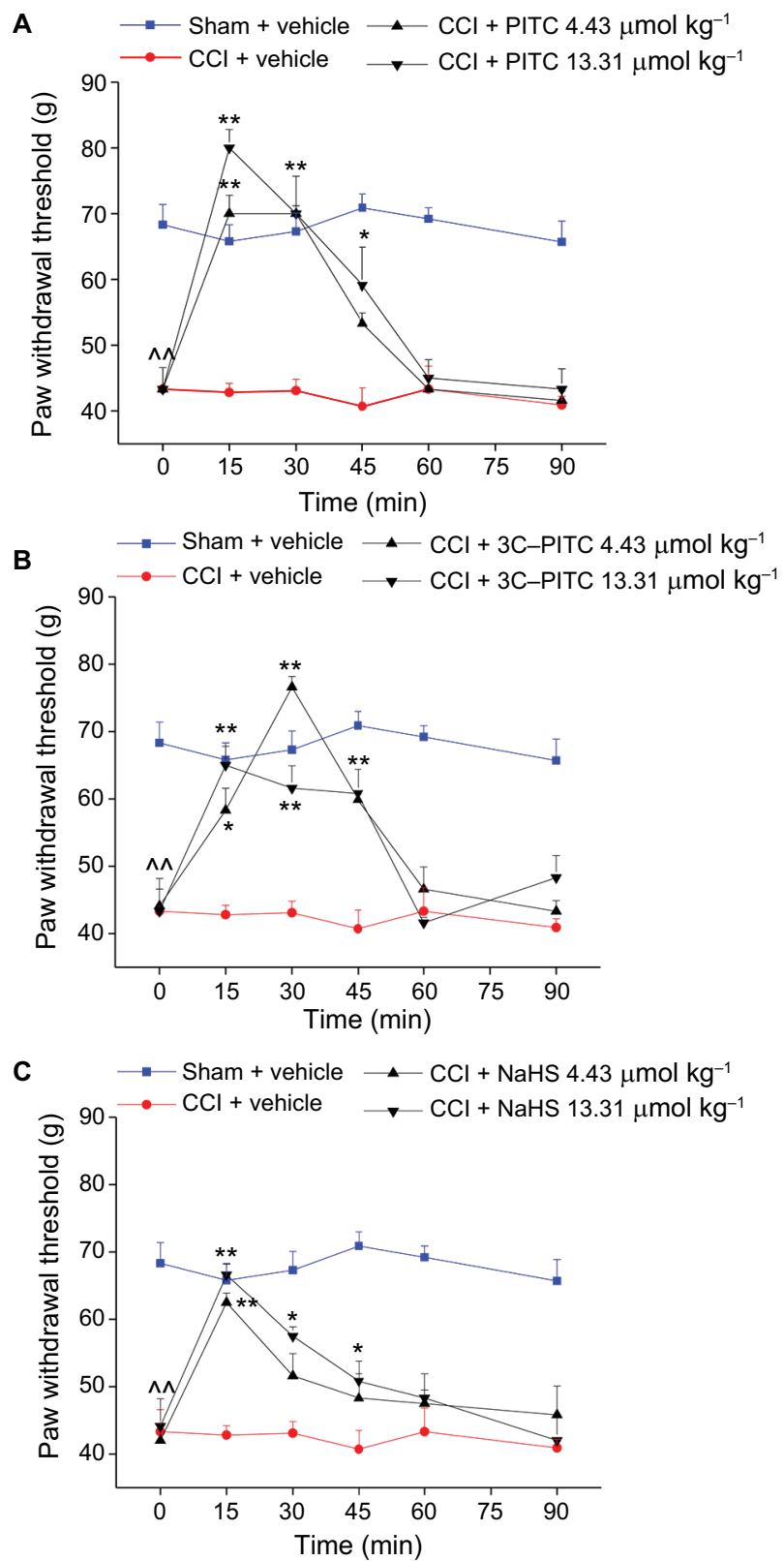

Figure I Effects of PITC, 3C-PITC, and NaHS on chronic constriction injury (CCl)induced mechanical hypersensitivity.

Notes: Mononeuropathy was induced by ligation of the right sciatic nerve $(\mathrm{CCl})$. Control animals were sham-operated and treated with vehicles. Mechanical hypersensitivity was evaluated by paw-pressure test, and data are expressed as the weight (g) that the animal tolerated on the paw. On day I4 after surgery, PITC (A), 3C-PITC (B), and NaHS (C) were each administered subcutaneously at doses of 4.43 and $13.31 \mu \mathrm{mol} \mathrm{kg}{ }^{-1}$ and measurements assessed before and 15, 30, 45, 60 and 90 minutes after injection. Each value represents the mean of ten rats per group, performed in two different experimental sets. ${ }^{\wedge} P<0.0$ I vs controls (sham + vehicletreated rats); $* P<0.05, * * P<0.01$ vs $C C l+$ vehicle-treated rats. Abbreviations: PITC, phenyl ITC; 3C-PITC, 3-carboxyphenyl ITC. 
CCI animals between 15 and 30 minutes after administration, and the effect of $13.31 \mu \mathrm{mol} \mathrm{kg} \mathrm{kg}^{-1}$ PITC lasted until 45 minutes (Figure 1A). Both doses of 3C-PITC were active between 15 and 45 minutes: $4.43 \mu \mathrm{mol} \mathrm{kg}{ }^{-1} 3 \mathrm{C}$-PITC peaked at 30 minutes, while the effect of the higher dose plateaued from 15 minutes and lasted up to 45 minutes (Figure 1B). The effect of NaHS was dose-dependent, with a maximum at 15 minutes after administration for both doses (Figure 1C).

In Figure 2, the acute effects of two different doses of PITC, 3C-PITC and NaHS on CCI-induced hind-limb weight-bearing alterations (measured by incapacitance test) are shown. On day 14 after operation, the difference between the weight burden on the contralateral and ipsilateral limbs ( $\Delta$ weight) was significantly increased in $\mathrm{CCI}+$ vehicle $(61.9 \pm 1.3 \mathrm{~g})$ with respect to sham + vehicle $(5.3 \pm 1.2$ $\mathrm{g}$, Figure 2). Both doses of PITC significantly decreased $\Delta$ weight $(\sim 50 \%)$ between 15 and 30 minutes after administration. The effect of $4.43 \mu \mathrm{mol} \mathrm{kg} \mathrm{kg}^{-1}$ PITC lasted longer, being significant even at 60 minutes (Figure 2A). Following the administration of both doses of $3 \mathrm{C}$-PITC, CCI-induced postural imbalance was reduced by $\sim 60 \%$ (Figure $2 \mathrm{~B}$ ). The lower dose was active between 15 and 45 minutes, while the effect of the higher dose began 30 minutes after administration. Both doses of NaHS reduced $\Delta$ weight by $\sim 50 \%$. The effect of $4.43 \mu \mathrm{mol} \mathrm{kg} \mathrm{kg}^{-1} \mathrm{NaHS}$ was maximal between 30 and 45 minutes, whereas the effect of the higher dose showed a peak 15 minutes after administration (Figure 2C).

The pharmacological activity of PITC, 3C-PITC, and NaHS (SC administration) was also evaluated in the rat unilateral osteoarthritis model induced by MIA. On day 14 after intra-articular MIA injection, the weight tolerated by the animals on the ipsilateral paw was significantly reduced $(42.5 \pm 3.5 \mathrm{~g})$ compared to control rats $(73.2 \pm 2.4 \mathrm{~g}$;

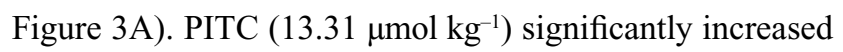
pain threshold between 15 and 45 minutes after administration, while the lower dose was less effective and delayed over time (Figure 3A). The effect of 3C-PITC was dose-dependent: both dosages produced a maximum effect 15 minutes after administration that lasted until 45 minutes (Figure 3B). The higher dose of 3C-PITC reverted mechanical hypersensitivity induced by MIA injection. Both doses of NaHS were active between 15 and 45 minutes $(60 \pm 5.7 \mathrm{~g}$ and $56.6 \pm 0.8 \mathrm{~g}$ for 4.43 and $13.31 \mu \mathrm{mol} \mathrm{kg} \mathrm{kg}^{-1}$, respectively; Figure $3 \mathrm{C}$ ).

In Figure 4, the effects of PITC, 3C-PITC, and NaHS on MIA-induced hind-limb weight-bearing alterations are shown. On day 14 after intra-articular MIA injection, $\Delta$ weight

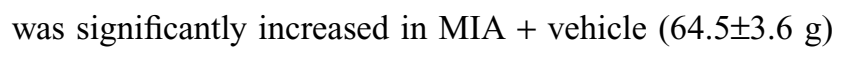
with respect to vehicle + vehicle (10.4 $\pm 1.3 \mathrm{~g}$; Figure 4A).
Both doses of PITC and 3C-PITC were equally active, decreasing $\Delta$ weight by $\sim 50 \%$ between 15 and 45 minutes (Figure 4A and B). Both doses of NaHS were able to reduce $\Delta$ weight by $\sim 50 \%$ : the lower dose was active between 30 and 45 minutes and the higher dose between 15 and 30 minutes (Figure 4C).

\section{Discussion}

From the present results, the preclinical efficacy of acute administration of ITCs or NaHS against neuropathic and osteoarthritic pain was proven. PITC and 3C-PITC were able to completely revert alterations in pain thresholds induced by CCI nerve surgery and MIA intra-articular injection. Neuropathic pain manifestations include spontaneous (tingling, burning, electric shock-like) pain, dysesthesia, paresthesia, allodynia (pain resulting from normally unpainful stimuli), and hyperalgesia (an increased response to painful stimuli). ${ }^{29}$ Its impact on people's health is strong, leading to the loss of work ability and becoming a social and economic burdens. ${ }^{30}$ Current pharmacological treatments for neuropathic pain, which include tricyclic antidepressants, serotonin-reuptake inhibitors, anticonvulsants, and opioids, have limited efficacy and a considerable number of side effects. ${ }^{5}$ The animal model of nerve CCI developed by Bennett and Xie reproduces many features of neuropathic pain disorders that occur in humans. ${ }^{23,31}$ Animals show altered spontaneous behavior consistent with the presence of ongoing neuropathic pain, lowered thresholds, and exaggerated responses to thermal and mechanical stimuli. ${ }^{32,33}$ Therefore, this model can be effectively used to study the efficacy of novel treatments for neuropathic pain.

Osteoarthritis is likewise characterized by persistent pain with inflammatory and neuropathic components, whose most common symptoms are stiffness and increasing pain at rest and in the chronic phase hyperalgesia. ${ }^{6,731}$ In particular, this pathology is characterized by a progressive loss of articular cartilage, new bone formation, and synovial proliferation, which result in inflammation, loss of joint function, and pain. The progressive damage of peripheral nerve ends evokes a neuropathic component that contributes to pain chronicity. ${ }^{6,34,35}$ The injection of MIA into the intra-articular space of the knee is an established and well-characterized preclinical model of osteoarthritis consistent with human pathophysiology and clinical symptoms..$^{34,36,37}$

Both CCI- and MIA-induced pain models present an initial relevant inflammatory condition that gradually evolves in neuropathic aspects. ${ }^{35,38}$ Since the therapies currently in use for neuropathic and osteoarthritic pain 

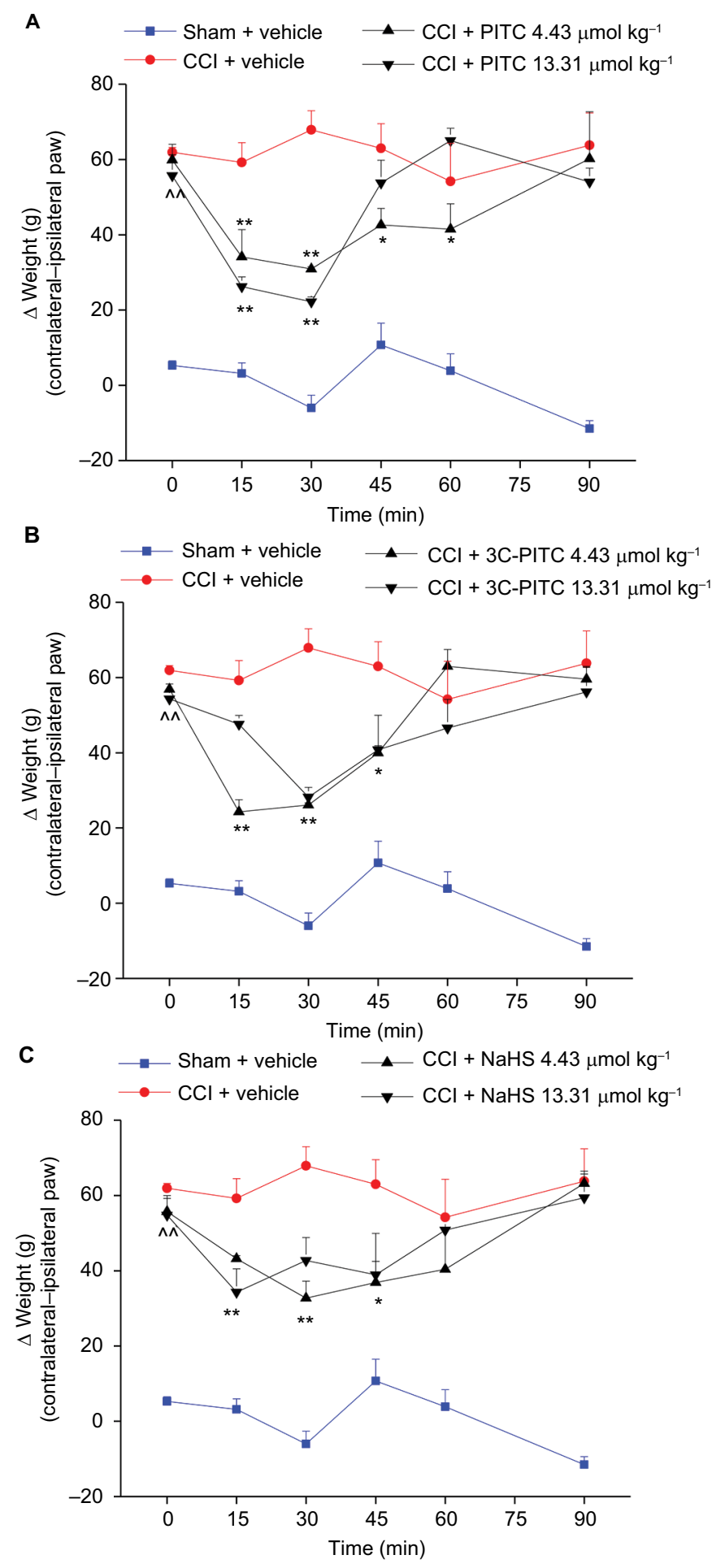

Figure 2 Effects of PITC, 3C-PITC, and NaHS on chronic constriction injury (CCl)-induced postural imbalance.

Notes: Mononeuropathy was induced by ligation of the right sciatic nerve (CCl). Control animals were sham-operated and treated with vehicles. Weight-bearing changes were evaluated by incapacitance test and data are expressed as the difference ( $\Delta$ weight) between the weight applied on the limb contralateral to the injury and the weight applied on the ipsilateral one. On day I4 after animal operation, PITC (A), 3C-PITC (B), and NaHS (C) were each administered subcutaneously at doses of 4.43 and I3.3I $\mu \mathrm{mol} \mathrm{kg}{ }^{-1}$ and measurements assessed before treatment and 15, 30, 45, 60 and 90 minutes after injection. Each value represents the mean of ten rats per group, performed in two different experimental sets. $\wedge \wedge P<0.01$ vs sham + vehicle-treated animals; $* P<0.05, * * P<0.01$ with respect to the pretest. Abbreviations: PITC, phenyl isothiocyanate; 3C-PITC, 3-carboxyphenyl ITC. 

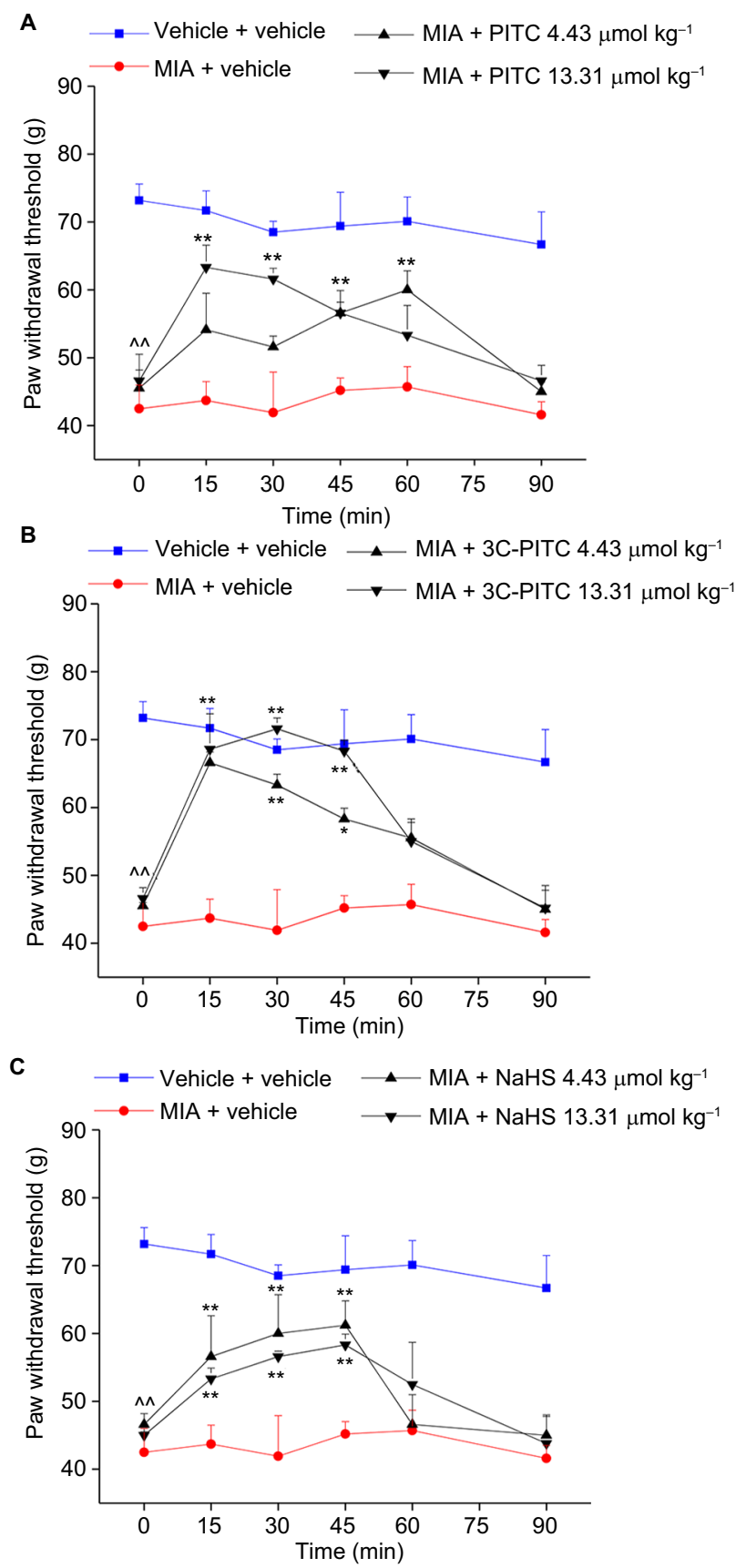

Figure 3 Effects of PITC, 3C-PITC, and NaHS on monoiodoacetate (MIA)-induced mechanical hypersensitivity.

Notes: Monoarthritis was induced by injection of MIA ( $2 \mathrm{mg} / 25 \mu \mathrm{L}$ saline solution) in the right (ipsilateral) articular cavity. The left (contralateral) joint was injected with the same volume of saline. Mechanical hypersensitivity was evaluated by paw-pressure test and data expressed as the weight $(\mathrm{g})$ that the animal tolerated on the paw. On day I4, PITC (A), 3C-PITC (B), and NaHS (C) were each administered subcutaneously at doses of 4.43 and I3.3I $\mu$ mol kg-1 and measurements assessed before treatment and I5, 30, 45, 60 and 90 minutes after injection. Each value represents the mean of ten rats per group, performed in two different experimental sets. ${ }^{\wedge} P<0.0 \mathrm{I}$ vs vehicle + vehicle-treated animals; $* P<0.05, * * P<0.01$ vs $M I A+$ vehicle-treated rats.

Abbreviations: PITC, phenyl isothiocyanate; 3C-PITC, 3-carboxyphenyl ITC.

have so far been unsatisfactory, the good activity and high potency presently shown by ITCs is noteworthy. PITC and 3C-PITC are $\mathrm{H}_{2} \mathrm{~S}$-releasing compounds, and the involvement of this gasotransmitter in their antinociceptive effects has already been demonstrated. ${ }^{16,21,22}$ The prototypical $\mathrm{H}_{2} \mathrm{~S}$ donor NaHS showed similar efficacy to ITCs, despite being less potent, probably because of the faster $\mathrm{H}_{2} \mathrm{~S}$-release kinetic. ITCs and NaHS also reduce postural imbalance, a feature of neuropathy progression that occurs in both models of mono-lateral neuropathy. ${ }^{23,28}$ This measure assesses the somatosensory component, highlighting spontaneous, unevoked pain. ${ }^{7}$ 

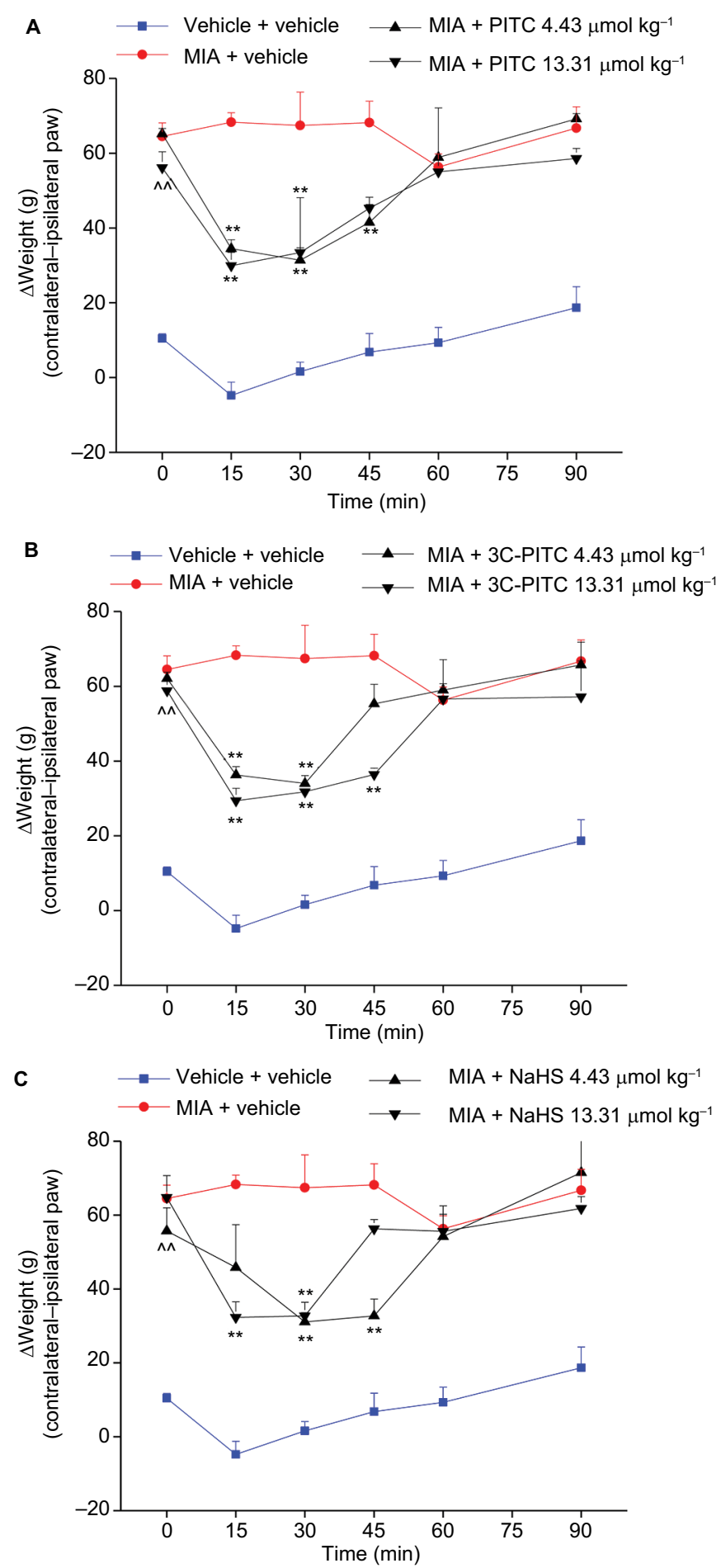

Figure 4 Effects of PITC, 3C-PITC, and NaHS on monoiodoacetate (MIA)-induced postural imbalance.

Notes: Monoarthritis was induced by injection of MIA $(2 \mathrm{mg} / 25 \mu \mathrm{L}$ saline solution) in the right (ipsilateral) articular cavity. The left (contralateral) joint was injected with the same volume of saline. Weight-bearing changes were evaluated by incapacitance test and data expressed as the difference ( $\Delta$ weight) between the weight applied on the limb contralateral to the injury and the weight applied on the ipsilateral one. On day I4, PITC (A), 3-C-PITC (B), and NaHS (C) were each administered subcutaneously at doses of 4.43 and $13.31 \mu \mathrm{mol} \mathrm{kg}$ and measurements assessed before treatment and I5, 30, 45, 60 and 90 minutes after injection. Each value represents the mean of ten rats per group, performed in two different experimental sets. ${ }^{\wedge \wedge} P<0.01$ vs vehicle + vehicle-treated animals; ${ }^{* *} P<0.01$ with respect to the pretest.

Abbreviations: PITC, phenyl isothiocyanate; 3C-PITC, 3-carboxyphenyl ITC. 
In accordance with our results, several studies have suggested significant pain-relieving effects of $\mathrm{NaHS}$ and other $\mathrm{H}_{2} \mathrm{~S}$ donors against neuropathic and visceral pain. ${ }^{39-41} \mathrm{On}$ the other hand, the beneficial effects of ITCs in models of central and peripheral nervous tissue injury and their efficacy in inflammatory states have been shown. ${ }^{11-14}$ In particular, their anti-inflammatory properties were assigned to the regulation of NF $\mathrm{B}$, modulation of TNF $\alpha$, and lipopolysaccharidestimulated inflammatory response. ${ }^{17-20}$

It is important to note that PITC, 3C-PITC, and NaHS show pain-relieving efficacy after acute systemic administration of very low doses. Furthermore, these compounds reach their maximum effect very quickly. This last property could be very useful in the treatment of acute pain attacks in patients with chronic pain conditions. Furthermore, their anti-inflammatory and antioxidative properties could offer further advantages in reducing disease progression by repeated treatments.

As a whole, the results gathered encourage us to continue to investigate the efficacy of these compounds in pain therapy. In particular, our efforts will be oriented to improve the pharmacokinetic profile of these compounds to make their effect longer-lasting. On the other hand, our recent in vivo data demonstrating that the antinociceptive effects of ITC-based compounds are mediated by their capacity of $\mathrm{H}_{2} \mathrm{~S}$ release, ${ }^{21}$ suggest that more persistent effects could be obtained by the modulation of $\mathrm{H}_{2} \mathrm{~S}$ release from ITCs or by the identification of ITCs with a slow $\mathrm{H}_{2} \mathrm{~S}$-release profile. In fact, the effects displayed by this gasotransmitter show rapid kinetics, both in vivo and in vitro: $\mathrm{H}_{2} \mathrm{~S}$ is able to reach the bloodstream easily and then its molecular targets on the cell membrane, inside the cytosol, or in intracellular organelles. ${ }^{42}$ From this perspective, the increase in ITC-effect duration would also be achieved by the application of sustained-release dosage forms.

\section{Conclusion}

Overall, this evidence, along with the data reporting the effectiveness of ITCs also in chemotherapy-induced neuropathic pain, can lead to a new and innovative strategy to treat traditional drug-resistant forms of pain, particularly in view of ITCs effectiveness and potency. ${ }^{21}$

\section{Acknowledgments}

This research was supported by the Italian Ministry of Instruction, University, and Research (MIUR), the University of Florence, and the University of Pisa.

\section{Disclosure}

The authors report no conflicts of interest in this work.

\section{References}

1. Goldberg DS, Mcgee SJ. Pain as a global public health priority. $B M C$ Public Health. 2011;11(1):770.

2. Neogi T. The epidemiology and impact of pain in osteoarthritis. Osteoarthritis Cartilage. 2013;21(9):1145-1153.

3. van Hecke O, Austin SK, Khan RA, Smith BH, Torrance N. Neuropathic pain in the general population: a systematic review of epidemiological studies. Pain. 2014;155(4):654-662.

4. Treede RD, Jensen TS, Campbell JN, et al. Neuropathic pain: redefinition and a grading system for clinical and research purposes. Neurology. 2008;70(18):1630-1635.

5. Finnerup NB, Attal N, Haroutounian S, et al. Pharmacotherapy for neuropathic pain in adults: a systematic review and meta-analysis. Lancet Neurol. 2015;14(2):162-173.

6. Gwilym SE, Keltner JR, Warnaby CE, et al. Psychophysical and functional imaging evidence supporting the presence of central sensitization in a cohort of osteoarthritis patients. Arthritis Rheum. 2009;61(9):1226-1234.

7. Liu P, Okun A, Ren J, et al. Ongoing pain in the MIA model of osteoarthritis. Neurosci Lett. 2011;493(3):72-75.

8. Crofford LJ. Use of NSAIDs in treating patients with arthritis. Arthritis Res Ther. 2013;15(Suppl 3):S2.

9. Kashfi K, Olson KR. Biology and therapeutic potential of hydrogen sulfide and hydrogen sulfide-releasing chimeras. Biochem Pharmacol. 2013;85(5):689-703.

10. Dinkova-Kostova AT, Kostov RV. Glucosinolates and isothiocyanates in health and disease. Trends Mol Med. 2012;18(6):337-347.

11. Giacoppo S, Galuppo M, de Nicola GR, Iori R, Bramanti P, Mazzon E. $4(\alpha-1$-rhamnosyloxy)-benzyl isothiocyanate, a bioactive phytochemical that attenuates secondary damage in an experimental model of spinal cord injury. Bioorg Med Chem. 2015;23(1):80-88.

12. Benedict AL, Mountney A, Hurtado A, et al. Neuroprotective effects of sulforaphane after contusive spinal cord injury. $J$ Neurotrauma. 2012;29(16):2576-2586.

13. Toyama T, Shinkai Y, Yasutake A, Uchida K, Yamamoto M, Kumagai Y. Isothiocyanates reduce mercury accumulation via an Nrf2-dependent mechanism during exposure of mice to methylmercury. Environ Health Perspect. 2011;119(8):1117-1122.

14. Innamorato NG, Rojo AI, García-Yagüe AJ, Yamamoto M, de Ceballos ML, Cuadrado A. The transcription factor Nrf2 is a therapeutic target against brain inflammation. J Immunol. 2008;181(1):680-689.

15. Karmakar S, Weinberg MS, Banik NL, Patel SJ, Ray SK. Activation of multiple molecular mechanisms for apoptosis in human malignant glioblastoma T98G and U87MG cells treated with sulforaphane. Neuroscience. 2006;141(3):1265-1280.

16. Martelli A, Testai L, Citi V, et al. Pharmacological characterization of the vascular effects of aryl isothiocyanates: is hydrogen sulfide the real player? Vascul Pharmacol. 2014;60(1):32-41.

17. Heiss E, Herhaus C, Klimo K, Bartsch H, Gerhäuser C. Nuclear factor kappa $\mathrm{B}$ is a molecular target for sulforaphane-mediated antiinflammatory mechanisms. J Biol Chem. 2001;276(34):32008-32015.

18. Rose P, Won YK, Ong CN, Whiteman M. Beta-phenylethyl and 8-methylsulphinyloctyl isothiocyanates, constituents of watercress, suppress LPS induced production of nitric oxide and prostaglandin E2 in RAW 264.7 macrophages. Nitric Oxide. 2005;12(4):237-243.

19. Xu C, Shen G, Chen C, Gélinas C, Kong AN. Suppression of NF-kappaB and NF-kappaB-regulated gene expression by sulforaphane and PEITC through IkappaBalpha, IKK pathway in human prostate cancer PC-3 cells. Oncogene. 2005;24(28):4486-4495.

20. Dey M, Ribnicky D, Kurmukov AG, Raskin I. In vitro and in vivo anti-inflammatory activity of a seed preparation containing phenethylisothiocyanate. J Pharmacol Exp Ther. 2006;317(1):326-333.

21. di Cesare Mannelli L, Lucarini E, Micheli L, et al. Effects of natural and synthetic isothiocyanate-based $\mathrm{H}_{2} \mathrm{~S}$-releasers against chemotherapyinduced neuropathic pain: Role of Kv7 potassium channels. Neuropharmacology. 2017;121:49-59. 
22. Citi V, Martelli A, Testai L, Marino A, Breschi MC, Calderone V. Hydrogen sulfide releasing capacity of natural isothiocyanates: is it a reliable explanation for the multiple biological effects of Brassicaceae? Planta Med. 2014;80(8-9):610-613.

23. Bennett GJ, Xie YK. A peripheral mononeuropathy in rat that produces disorders of pain sensation like those seen in man. Pain. 1988;33(1):87-107.

24. Guingamp C, Gegout-Pottie P, Philippe L, Terlain B, Netter P, Gillet P. Mono-iodoacetate-induced experimental osteoarthritis. Arthritis Rheum. 1977;40:1670-1679.

25. Martelli A, Testai L, Breschi MC, et al. Vasorelaxation by hydrogen sulphide involves activation of Kv7 potassium channels. Pharmacol Res. 2013;70(1):27-34.

26. Mcgrath JC, Lilley E. Implementing guidelines on reporting research using animals (ARRIVE etc.): new requirements for publication in BJP. Br J Pharmacol. 2015;172(13):3189-3193.

27. Leighton GE, Rodriguez RE, Hill RG, Hughes J. kappa-Opioid agonists produce antinociception after i.v. and i.c.v. but not intrathecal administration in the rat. Br J Pharmacol. 1988;93(3):553-560.

28. Bove SE, Calcaterra SL, Brooker RM, et al. Weight bearing as a measure of disease progression and efficacy of anti-inflammatory compounds in a model of monosodium iodoacetate-induced osteoarthritis. Osteoarthritis Cartilage. 2003;11(11):821-830.

29. Jensen TS, Finnerup NB. Allodynia and hyperalgesia in neuropathic pain clinical manifestations and mechanisms. Lancet Neurol. 2014;13(9): 924-935.

30. Jensen TS, Baron R, Haanpää M, et al. A new definition of neuropathic pain. Pain. 2011;152(10):2204-2205.

31. Woolf CJ, Mannion RJ. Neuropathic pain: aetiology, symptoms, mechanisms, and management. Lancet. 1999;353(9168):1959-1964.

32. Attal N, Jazat F, Kayser V, Guilbaud G. Further evidence for 'painrelated' behaviours in a model of unilateral peripheral mononeuropathy. Pain. 1990;41(2):235-251.
33. Tal M, Bennett GJ. Extra-territorial pain in rats with a peripheral mononeuropathy: mechano-hyperalgesia and mechano-allodynia in the territory of an uninjured nerve. Pain. 1994;57(3):375-382.

34. Ivanavicius SP, Ball AD, Heapy CG, Westwood FR, Murray F, Read SJ. Structural pathology in a rodent model of osteoarthritis is associated with neuropathic pain: increased expression of ATF-3 and pharmacological characterisation. Pain. 2007;128(3):272-282.

35. Oteo-Álvaro Á, Ruiz-Ibán MA, Miguens X, Stern A, Villoria J, Sánchez-Magro I. High Prevalence of Neuropathic Pain Features in Patients with Knee Osteoarthritis: A Cross-Sectional Study. Pain Pract. 2015;15(7):618-626.

36. Pomonis JD, Boulet JM, Gottshall SL, et al. Development and pharmacological characterization of a rat model of osteoarthritis pain. Pain. 2005;114(3):339-346.

37. Chandran P, Pai M, Blomme EA, Hsieh GC, Decker MW, Honore P. Pharmacological modulation of movement-evoked pain in a rat model of osteoarthritis. Eur J Pharmacol. 2009;613(1-3):39-45.

38. Daemen MA, Kurvers HA, Kitslaar PJ, Slaaf DW, Bullens PH, van den Wildenberg FA. Neurogenic inflammation in an animal model of neuropathic pain. Neurol Res. 1998;20(1):41-45.

39. Distrutti E, Sediari L, Mencarelli A, et al. Evidence that hydrogen sulfide exerts antinociceptive effects in the gastrointestinal tract by activating KATP channels. J Pharmacol Exp Ther. 2006;316(1):325-335.

40. Lin JQ, Luo HQ, Lin CZ, Chen JZ, Lin XZ. Sodium hydrosulfide relieves neuropathic pain in chronic constriction injured rats. Evid Based Complement Alternat Med. 2014;2014:1-7.

41. Kida K, Marutani E, Nguyen RK, Ichinose F. Inhaled hydrogen sulfide prevents neuropathic pain after peripheral nerve injury in mice. Nitric Oxide. 2015;46:87-92.

42. Wallace JL, Wang R. Hydrogen sulfide-based therapeutics: exploiting a unique but ubiquitous gasotransmitter. Nat Rev Drug Discov. 2015;14(5):329-345.

\section{Journal of Pain Research}

\section{Publish your work in this journal}

The Journal of Pain Research is an international, peer reviewed, open access, online journal that welcomes laboratory and clinical findings in the fields of pain research and the prevention and management of pain. Original research, reviews, symposium reports, hypothesis formation and commentaries are all considered for publication

\section{Dovepress}

The manuscript management system is completely online and includes a very quick and fair peer-review system, which is all easy to use. Visit http://www.dovepress.com/testimonials.php to read real quotes from published authors. 